\title{
Malnutrition in Obesity: Is It Possible?
}

\author{
Milena Kobylińska Katarzyna Antosik Agnieszka Decyk Katarzyna Kurowska
}

Faculty of Medical and Health Sciences, Siedlce University of Natural Sciences and Humanities, Siedlce, Poland

\begin{abstract}
Keywords
Obesity · Malnutrition - Microbiota - Bariatric surgery ·

Adults
\end{abstract}

\begin{abstract}
Background: The World Health Organization (WHO) classifies malnutrition as the biggest threat to public health worldwide, and this condition is observed in $20-60 \%$ of hospitalized patients. Malnutrition is a state of the body in which due to insufficient supply or incorrect absorption of essential nutrients, the body composition changes and the body's functions are impaired. Malnutrition is associated not only with reduced body mass index but also with obesity. Summary: Obesity is defined as a paradoxical state of malnutrition, which despite excessive energy consumption is associated with a shortage of individual microelements. Deficiency or lack of homeostasis of essential micronutrients can significantly affect daily performance, intellectual and emotional state, but also the physical state of the body. Food deficiency can also contribute to further weight gain or the development of other metabolic diseases. Micronutrient deficiency may include not only incorrect dietary choices and insufficient access to nutrient-rich foods but also changes in the absorption, distribution or excretion of nutrients, and altered micronutrient metabolism resulting from systemic inflammation caused by obesity. An effective therapy method recommended for people with morbid obesity is bariatric surgery aimed at both weight loss and improving quality of
\end{abstract}

life. Unfortunately, the effects of these treatments are often medium- and long-term complications associated with micronutrient deficiency as a result of reduced consumption or absorption. Therefore, the use of bariatric surgery in patients with extreme obesity can affect the metabolism of microelements and increase the risk of nutritional deficiencies. Key Messages: Studies by many authors indicate a higher incidence of food deficiency among people with excessive body weight, than in people with normal body weight of the same age and same sex. Monitoring the concentration of minerals and vitamins in blood serum is a good practice in the treatment of obesity. The proper nutritional status of the body affects not only the state of health but also the effectiveness of therapy. The aim of the review was to present the issue of malnutrition in the context of obesity.

(c) 2021 The Author(s).

Published by S. Karger AG, Basel

\section{Introduction}

Malnutrition is a condition in which a deficiency of one or many nutrients has an adverse effect on cells, tissues, organs, and the body understood as a whole, which is manifested in the deterioration of its functioning and a negative change in the overall clinical picture [1]. The European Society for Clinical Nutrition and Metabolism (ESPEN) defines malnutrition as a state of the body in which due to insufficient supply or incorrect absorption
C 2021 The Author(s).

Published by S. Karger AG, Basel

This is an Open Access article licensed under the Creative Commons Attribution-NonCommercial-4.0 International License (CC BY-NC) (http://www.karger.com/Services/OpenAccessLicense), applicable to the online version of the article only. Usage and distribution for commercial purposes requires written permission.
Correspondence to:

Katarzyna Antosik, katarzyna.antosik@uph.edu.pl 
of essential nutrients, body composition changes, physical and mental impairment of the body and adverse effects on the outcome of the underlying disease [2]. The World Health Organization (WHO) classifies malnutrition as the biggest threat to public health worldwide, and this condition is observed in $20-60 \%$ of hospitalized patients $[3,4]$. Malnutrition is both medically and economically harmful. If detected early and treated appropriately, better clinical results and lower costs will be achieved. Malnutrition is an independent risk factor for patient morbidity and mortality and is associated with increased healthcare costs [1]. Malnutrition is associated not only with reduced body mass index, but also with obesity, in which adipose tissue is also a source of inflammatory cytokines [5]. Research suggests that the inflammatory process in the hypothalamus and mediators released at that time may also lead to a positive energy balance and, consequently, to the development of obesity. In experiments carried out on the animal model of obesity, a significant increase in inflammatory cytokine levels (tumor necrosis factor- $\alpha$, interleukin- $1 \beta$, and interleukin-6) was observed both in adipose tissue and in the hypothalamus [6-8]. According to WHO, obesity is "pathological accumulation of body fat, exceeding its physiological needs and adaptability" [9]. Causes of obesity can result from various hormonal, genetic, and metabolic processes, but obesity is also a condition resulting from lifestyle factors, including excessive food consumption or insufficient physical activity [10]. Obesity increases the risk of other disease entities, referred to as concomitant diseases, which include, but are not limited to, diabetes, hypertension, tumors (in particular colorectal cancer), osteoarthritis, or lipid disorders $[11,12]$.

\section{Data Sources and Searches}

A literature search using PubMed and Google Scholar was performed using the following terms in various combinations: obesity, malnutrition, microbiota, bariatric, and adults. The search for literature was conducted in the period from November 30,2020, to December 7, 2020, by all authors separately. Researchers used the same search words in both databases on different days to ensure that the search results were repeatable. All articles have been checked by title, abstract, and full text. Relevant articles have been reviewed and posted here. Language restrictions were not applied, but articles for which the full-text version could not be obtained were excluded.

\section{Nutritional Deficiencies in the Diet and Serum Levels of Nutrients in Patients with Obesity}

Diagnosing malnutrition in people with obesity is quite difficult. To determine the occurrence of malnutrition in a patient, an assessment of nutrition and nutritional status should be performed [13]. The most common methods of dietary assessment are 24/48-h dietary recall, ffq. Identification of malnutrition is also based on anthropometric measurements, blood biochemical tests, and physical parameters. An important element of assessing nutritional status and diet is dietary assessment consumed by people with obesity. This allows for early diagnosis and implementation of dietary treatment. Adequate supply of nutrients is particularly important when implementing a low-energy diet [13]. Studies by many authors [14-17] indicate that obesity is very often associated with the nutrition and qualitative malnutrition of the body. It is defined as a paradoxical state of malnutrition, which despite excessive energy consumption is associated with a shortage of individual microelements. Deficiency or lack of homeostasis of essential micronutrients can significantly affect daily performance, intellectual, and emotional state, but also the physical state of the body $[18,19]$. Food deficiency can also contribute to further weight gain or the development of other metabolic diseases [20]. Nutrient deficiencies associated with obesity may be partly due to overconsumption of foods that are high in calories but have low-nutrient densities [21]. This phenomenon is most common in highly developed countries. For example, in children and adults living in the USA, about $27-30 \%$ of total daily calorie intake comes from low-nutrient foods, mainly from added sugars and desserts [22]. In turn, Astrup and Bügel [23] report that a diet in which $>30 \%$ of energy demand is fat is characterized by a lower intake of vitamins $\mathrm{A}$ and $\mathrm{C}$ and folic acid. Micronutrient deficiency may include not only incorrect dietary choices, insufficient access to nutrientrich foods, but also changes in the absorption, distribution, or excretion of nutrients, and altered micronutrient metabolism resulting from systemic inflammation caused by obesity $[15,16,24]$. Studies by many authors indicate a higher incidence of food deficiency among people with excessive body weight, than in people with normal body weight of the same age and same sex [16, $25,26]$. Higher body weight resulting from increased body fat may result in lower serum vitamin $\mathrm{D}_{3}$ levels. Vitamin $\mathrm{D}_{3}$ deficiency in people with obesity may be associated with reduced physical activity, thus less exposure to the sun and increased storage of vitamin $\mathrm{D}_{3}$ in adipose 
tissue, where it is not available to regulate calcium metabolism [21, 27]. Deficiency of B vitamins is also observed in people with obesity [28]. Low levels of thiamine were found in $15-29 \%$ of people with obesity [29]. Higher body mass index is a significant clinical risk of thiamine deficiency. The explanatory mechanisms for the development of thiamine deficiency in people with obesity remain unclear. There are no data indicating that thiamine supplementation brings any clinical benefits in patients with excessive body weight [30]. While the mechanisms of thiamine deficiency in obese individuals remain unclear, there is some speculation that thiamine deficiency in obese individuals is caused by a diet high in simple sugars. These products are not only thiaminefree, but the metabolism of sugar-rich foods also requires relatively large amounts of thiamine, and therefore, consumption of these products may result in a deficiency of this vitamin [31]. In turn, abnormal levels of vitamin $B_{12}$ were observed in $11 \%$ of people with morbid obesity [ 17 , 32]. Vitamin $B_{12}$ supplementation has been shown to improve the deficiency rate to around $4 \%$, but the use of multivitamins alone is usually not enough to prevent deficiency [21]. In turn, Vitolo et al. [33] showed an increased risk of insufficient folic acid intake in adolescents with a waist circumference above the 80th percentile. Studies in Brazil and Spain have shown a folic acid deficiency in $54.3 \%$ of adolescents and adults with obesity [32]. It has also been shown that almost $50 \%$ of people with obesity have insufficient levels of vitamin C (defined as serum vitamin $\mathrm{C}$ below the lower limit of the laboratory norm $[<4.6 \mathrm{mg} / \mathrm{L}])[34]$ and that a higher $\mathrm{BMI}$ is correlated with lower serum vitamin C levels [35]. Prospective studies demonstrating the clinical benefit of vitamin C supplementation in patients with obesity are therefore needed [23]. In addition, studies in people with obesity have shown that low magnesium levels are correlated with diseases associated with chronic inflammation $[35,36]$, while further analyses are needed to determine the relationship between magnesium content and obesity [37]. Research conducted in the USA among children with obesity and normal body weight showed that serum alpha-tocopherol levels were significantly lower in obese children than in children with normal body weight [38]. Another study found that men and women who were overweight and obese had lower serum levels of vitamin $\mathrm{E}$ than those of normal weight: this study showed a correlation between BMI and vitamin E levels - the higher the BMI value, the lower the serum vitamin E level [39]. The observed low vitamin E levels among people with overweight and obese may result from increased systemic and adipose-specific oxidative stress for people with overweight, which may lead to increased oxidative catabolism of these fat-soluble nutrients [40, 41].

Astrup and Bügel [23] believe that along with recommendations for healthy eating and increased physical activity, supplementation with specific micronutrients or vitamins should be considered in people at highest risk or deficiencies. It should be noted that it is not recommended that increased intake of vitamins and minerals be based solely on food supplementation or fortification. Such strategies may discourage the use of healthy eating habits [42]. In addition, the bioavailability of nutrients in people with obesity is limited. Supplementation turns out not to be the best solution in the case of large nutritional deficiencies [19].

\section{Microbiota Dysfunction in People with Obesity}

Pilot studies conducted by Damms-Machado et al. [25] did not show an improvement in the levels of selected microelements after the introduction of a balanced low-calorie diet for people with obesity. The diet covered $100 \%$ of the demand for micronutrients in accordance with adult standards. The lack of a positive effect can be explained by the dysfunction of the intestinal microbiota in these people. Recently, it has been promoting the role of the intestinal microbiota in the pathogenesis of obesity. Gut bacteria are a source of lipopolysaccharide (LPS), which induces chronic inflammation. LPS is recognized by immunocytes through toll-like receptor 4 , to which it binds with high affinity, thereby stimulating immune cells to produce pro-inflammatory cytokines that trigger inflammation [43]. Increased LPS levels in plasma and intestines are observed in people with obesity. Intestinal barrier disorders in people with obesity may result from long-term poor eating habits [44]. Another area of research concerns the metabolic role of intestinal microbes in energy production, storage, and expenditure related to obesity. The results of studies suggest that the composition of the intestinal microflora is different in lean people with obesity and animals [16]. Studies on germ-free mice that have been transplanted with the intestinal bacteria of obese mice and mice with normal body weight have demonstrated the relationship between intestinal microflora and obesity. Using the same diet in both groups, it was observed that germ-free mice transplanted with excess weight mice also became obese. In addition, obese mice showed a 50\% decrease in the number of Bacteroides and a proportional increase in 
Firmicutes [45]. Composition, variety, and metabolic activity of gut microbiota (GM) are closely related to nutrient intake and dietary patterns. Specific dietary patterns and nutritional factors alter GM profiles that may regulate or influence the progression of obesity [46]. Many dietary patterns, such as the Western diet, vegan diet, vegetarian diet, gluten-free diet, and Mediterranean diet, have been shown to influence the marked diversity of the gut microflora that can affect the host metabolism [47]. However, more research is needed to understand the impact of dietary patterns on obesity-related GM changes [46]. Evidence linking gut bacteria to host metabolism could enable the development of new therapeutic strategies based on the modulation of the gut microflora to treat or prevent obesity [48]. In people with obesity, the ratio of Bacteroidetes to Firmicutes was also disturbed in favor of the latter. Weight reduction is associated with an increase in the number of Bacteroidetes bacteria proportional to the number of kilos lost [49]. It has been proven that a $20 \%$ increase in the number of Firmicutes and an analogous decrease in the number of Bacteroidetes are responsible for an increase by $150 \mathrm{kcal}$ of energy intake from food [50]. What is more, as a result of bariatric surgery (Roux-en-Y gastric bypass [RYGB] - bypass gastrointestinal anastomosis with Roux-en-Y loop), leading to weight loss in people with obesity, researchers observed an increase in the number of Bacteroidetes in them [51]. A study of pregnant women who are overweight and pregnant women who have normal weight showed that the composition of the microflora is different, with a proportionally higher number of Bacteroidetes and Staphylococcus in women who are overweight during pregnancy [52]. In addition, intestinal dysbiosis can alter the production of gastrointestinal peptides associated with satiety, resulting in increased food intake [53]. At this point, however, it should be mentioned that not all studies confirm such a significant contribution of the intestinal microbiota in the pathogenesis of obesity. Ley et al. [54] found identical numbers of Bacteroidetes and Firmicutes in people with obesity who were fed a high-fat diet after changing to a low-calorie diet. There have also been studies suggesting no relationship between Bacteroidetes/Firmicutes and diet and body mass index [55]. This topic still requires a lot of research and analysis, taking into account all possible factors that affect the credibility of the results [56]. The heterogeneity of the methods used to quantify the level of the gut microflora does not allow a proper comparison of the results obtained from different studies, as each technique is burdened with issues of accuracy, sensitivity, or specificity. There- fore, there is a need to standardize the techniques used to detect and classify the composition of the intestinal microflora in obese people [57]. Gender, age, genotype, and environmental conditions also affect the composition of the intestinal microbiota outside of the diet. Intestinal microbiota changes with age, as shown by comparing the microbiota of children, adults, and the elderly $[58,59]$. It is possible to directly modulate the intestinal microflora with probiotics, prebiotics, antibiotics, or other therapeutic interventions. While several randomized controlled trials have been conducted on probiotics in obesity, the results are not yet convincing. Therefore, there is a lack of more randomized, placebo-controlled in this regard [57].

\section{Bariatric Obesity Treatment and Nutritional Deficiencies}

An effective therapy method recommended for people with morbid obesity (BMI $\geq 40$ ) is surgical treatment of obesity, that is, bariatric surgery aimed at both weight loss and improving quality of life [60]. Unfortunately, the effects of these treatments are often medium- and longterm complications associated with micronutrient deficiency as a result of reduced consumption or absorption $[21,61]$. Therefore, the use of bariatric surgery in patients with extreme obesity can affect the metabolism of microelements and increase the risk of nutritional deficiencies [62]. The type of bariatric surgery performed may also affect the occurrence of nutritional deficiencies, especially of some microelements and macroelements, depending on which part of the gastrointestinal tract is bypassed [23]. A study by Guan et al. [63] showed that nutritional deficiencies were common in patients undergoing RYGB and sleeve gastrectomy. Vitamin $\mathrm{D}$ deficiency was the most severe (78.8\%), followed by vitamin $\mathrm{B}_{1}(39.2 \%)$, vitamin $\mathrm{B}_{6}(28.0 \%)$, folate $(26.8 \%)$, vitamin $\mathrm{C}(18.0 \%)$, albumin (13.4\%), transferrin (11.6\%), phosphorus (11.5\%), and vitamin $\mathrm{B}_{12}$ (3.3\%). One year after surgery, folate deficiency was found in $4 \%$ of RYGB patients and $10.8 \%$ of sleeve gastrectomy patients. The authors noted that despite postoperative routine multivitamin supplementation, the incidence of vitamin $B_{12}$ deficiency increased significantly in the RYGB group (16\%) 12 months after surgery. Additionally, preoperative vitamin $B_{12}$ levels were independently associated with postoperative decline, and preoperative vitamin $\mathrm{B}_{6}$ deficiency was a predictor of deficiency 1 year after surgery [64]. Newer procedures such as one anastomosis gastric bypass and single 
anastomosis duodenal ileostomy are likely to have better metabolic effects, but the risk of malnutrition and vitamin A deficiency is higher [65]. For example, iodine is absorbed in the stomach and small intestine [66]. Copper is absorbed in both the stomach and duodenum, while vitamin $B_{12}$ must bind to internal factors in the stomach before it is absorbed in the ileum. Other microelements, including iron, zinc, selenium, chromium, manganese, vitamins $\mathrm{A}, \mathrm{C}, \mathrm{E}$, and $\mathrm{K}$, thiamine, riboflavin, folic acid, niacin, biotin, pyridoxine, pantothenate, and calcium, are absorbed in the duodenum or jejunum [62]. According to Correia-Horvath et al. [67], food consumption before bariatric surgery in people with obesity is a factor that requires more careful examination, as it can help regain and maintain proper nutritional status in the postoperative period. Calorie intake in people with obesity is usually excessive, but there may be insufficient amounts of essential nutrients, leading to undiagnosed deficiencies. Studies indicate that nutritional deficiencies are observed in patients qualified for surgery before surgery (so-called preoperative deficiencies), which particularly affect iron, calcium, folic acid, vitamin $\mathrm{D}$, and vitamin $\mathrm{B}_{12}$ [68]. Iron deficiency may occur in patients with obesity before surgery due to constant inflammation. One of the basic mechanisms may be the production of hepcidin, which reduces serum iron levels. Bariatric operations may exacerbate this [69]. In addition to the above-mentioned components, deficiencies of vitamin A, copper, zinc, and selenium are also observed $[23,70,71]$. Thiamine deficiency in the range of $16-47 \%$ among patients with obesity undergoing bariatric surgery has also been demonstrated [72]. Observation and taking action to compensate for preoperative deficits is an important element in avoiding more serious complications after bariatric procedures [73].

\section{Summary}

Conducting research on the role of malnutrition and micronutrient deficiencies is important not only in the context of obesity, but also in the pathophysiology of comorbidities [17]. Actions aimed at reducing body weight should be undertaken with the greatest possible care, as they may be associated with a reduced intake of nutrients [19]. Monitoring the concentration of minerals and vitamins in blood serum is a good practice in the treatment of obesity. The proper nutritional status of the body affects not only the state of health, but also the effectiveness of therapy [13].

\section{Conclusion}

1. Before and during the implementation of diet therapy in patients with obesity, a comprehensive assessment of nutritional status should be performed. This examination should be performed by a specialist using subjective and objective parameters such as nutritional interview, clinical history, anthropometric measurements, physical parameters, and laboratory results. Systematic screening for nutritional risk and standardized nutritional management of people with obesity can also help reduce healthcare costs.

2. The obesity medical care plan should include a lifestyle change, choosing healthy, high-nutrient foods to prevent malnutrition. Fortified foods should also be included to prevent shortages. The use of specific vitamin supplements can be incorporated into this practice. The current recommended nutrition standards are standard, but it seems necessary to develop specific standards for people with obesity. There is a need to conduct further research explaining the factors shaping nutritional deficiencies in people who are overweight or obese, as well as appropriate treatment strategies to prevent them.

3. Physicians, dietetics, and nutritionists should be aware of potentially preexisting nutritional deficiencies in overweight or patients with obesity, as well as nutritional deficiencies following bariatric procedures. Observation and taking action to compensate for preoperative deficiencies is an important element in avoiding complications after bariatric surgeries.

4. The complex interaction of gut bacteria with the host should be elucidated, as well as the possible influence of variables such as diet, gender, age, and exercise. Future evidence may help by using the modulation of these variables to transform the gut microflora into a healthier profile.

5. Modifying the composition of the GM with a mixture of probiotic strains may be a promising strategy for treating obesity. However, further research is needed.

6. Further research should be carried out using standardized next-generation sequencing technologies on the actual association of gut microflora composition with specific obesity-related phenotypes.

\section{Conflict of Interest Statement}

The authors have no conflicts of interest to declare. 


\section{Funding Sources}

This work is funded by the Siedlce University of Natural Sciences and Humanities. There was no special funding for the writing of this paper, no grant from funding agencies in the public, commercial, or not-for-profit sectors.

\section{Author Contributions}

All authors provided substantial contribution to the design and implementation of this study and to the generation of the manuscript. The contributions of each author are as follows: M. Kobylińska and K. Antosik conceived the design and purpose of the work. M. Kobylińska, K. Antosik, A. Decyk, and K. Kurowska analyzed the data and interpreted the results based on the available literature. M. Kobylińska, K. Antosik, and A. Decyk drafted the manuscript. A. Decyk, K. Antosik, K. Kurowska, and M. Kobylińska provided critical revisions and final approval of the version submitted.

\section{References}

1 Löser C. Malnutrition in hospital: the clinical and economic implications. Dtsch Arztebl Int. 2010 Dec;107(51-52):911-7.

2 Burgos R, Bretón I, Cereda E, Desport JC, Dziewas R, Genton L, et al. ESPEN guideline clinical nutrition in neurology. Clin Nutr. 2018 Sep;37:354-96.

3 World Health Organization. Malnutrition. 2021 [cited 2021 May 7]. Available from: https://www.who.int/news-room/fact-sheets/ detail/malnutrition.

4 Ostrowska J, Jeznach-Steinhagen A. Fight against malnutrition (FAM) selected results of 2006-2012 nutrition day survey in Poland. Rocz Panstw Zakl Hig. 2016;67(3):291-300.

5 Gulland A. Malnutrition and obesity coexist in many countries, report finds. BMJ. 2016 Jun;353:i3351.

6 De Souza CT, Araujo EP, Bordin S, Ashimine R, Zollner RL, Boschero AC, et al. Consumption of a fat-rich diet activates a proinflammatory response and induces insulin resistance in the hypothalamus. Endocrinology. 2005 Oct;146(10):4192-9.

7 Zhang X, Zhang G, Zhang H, Karin M, Bai H, Cai D. Hypothalamic IKKbeta/NF-kappaB and ER stress link overnutrition to energy imbalance and obesity. Cell. 2008 Oct;135(1): 61-73.

8 Jais A, Brüning JC. Hypothalamic inflammation in obesity and metabolic disease. J Clin Invest. 2017 Jan;127(1):24-32.

9 World Health Organization. Obesity: preventing and managing the global epidemic, report of a WHO consultation. 2020 [cited 2020 Sep 7]. Available from: https://www. who.int/nutrition/publications/obesity/ WHO_TRS_894/en/.

10 ODPHP - Office of Disease Prevention and Health Promotion. Physical activity. 2020 [cited 2020 Sep 7]. Available from: www. healthypeople.gov/2020/topics-objectives/ topic/physical-activity.

11 Goossens GH. The metabolic phenotype in obesity: fat mass, body fat distribution, and adipose tissue function. Obes Facts. 2017 Jun; 10:207-15.

12 Polish Society of Dietetics. Recommendations of dietary treatment of obesity, position of the Polish Society of Dietetics. J Diet. 2019 Oct; 11:5-27.

13 Węgielska I, Słaba W, Suliburska J. Dietotherapy and pharmacotherapy of obesity in the aspect of malnutrition in the obese. For Zab Metab. 2011 Feb;2(4):239-44.

14 Vandevijvere S, Chow CC, Hall KD, Umali E, Swinburn BA. Increased food energy supply as a major driver of the obesity epidemic: a global analysis. Bull World Health Organ. 2015 Feb;93(7):446-56.

15 Bhatti O, Bielefeldt K, Nusrat S. Nutritional deficiencies in obesity and after weight reduction. Ann Nutr Disord \& The. 2015 Jul;2(2): $1-6$.

16 Gebler L, Charuvastra M, Silver D. Nutritional deficiencies associated with obesity. Obes Weight Loss Ther. 2015 Mar;5(2):1-4.

17 Sánchez A, Rojas P, Basfi-fer K, Carrasco F, Inostroza J, Codoceo J, et al. Micronutrient deficiencies in morbidly obese women prior to bariatric surgery. Obes Surg. 2016 Feb; 26(2):361-8.

18 Reynolds E. Vitamin B12, folic acid, and the nervous system. Lancet Neurol. 2006 Nov; 5(11):949-60.

19 Kaidar-Person O, Person B, Szomstein S, Rosenthal RJ. Nutritional deficiencies in morbidly obese patients: a new form of malnutrition? Part A: vitamins. Obes Surg. 2008 Jul; 18(7):870-6.

20 Muscogiuri G, Sorice GP, Prioletta A, Policola C, Della Casa S, Pontecorvi A, et al. 25-Hydroxyvitamin D concentration correlates with insulin-sensitivity and BMI in obesity. Obesity. 2010 Oct;18(10):1906-10.

21 Xanthakos SA. Nutritional deficiencies in obesity and after bariatric surgery. Pediatr Clin North Am. 2009 Oct;56(5):1105-21.

22 Kant AK. Reported consumption of low-nutrient-density foods by American children and adolescents: nutritional and health correlates, NHANES III, 1988 to 1994. Arch Pediatr Adolesc Med. 2003 Aug;157(8):78996.

23 Astrup A, Bügel S. Overfed but undernourished: recognizing nutritional inadequacies/ deficiencies in patients with overweight or obesity. Int J Obes. 2019 Feb;43(2):219-32.
24 Kubasik M, Bogdański P, Suliburska J. The influence of minerals in the pathogenesis of obesity and its complications. For Zab Metab. 2018 Feb;9(4):141-51.

25 Damms-Machado A, Weser G, Bischoff S. Micronutrient deficiency in obese subjects undergoing low calorie diet. Nutr J. 2012 Jun; $11(31): 1-10$.

26 Imoberdorf R, Ballmer PE. Die epidemiologie der mangelernährung. Ther Umsch. 2014 Feb;71:123-6.

27 Wąsowski M, Czerwińska E, MarcinowskaSuchowierska E. Obesity - the condition predisposing to vitamin D deficiency. Prog Med. 2012 Mar;25(3):258-64.

28 Poitou Bernert C, Ciangura C, Coupaye M, Czernichow S, Bouillot JL, Basdevant A. Nutritional deficiency after gastric bypass: diagnosis, prevention and treatment. Diabetes Metab. 2007 Feb;33(1):13-24.

29 Via M. The malnutrition of obesity: micronutrient deficiencies that promote diabetes. ISRN Endocrinol. 2012 Mar;2012:103472.

30 Nath A, Tran T, Shope TR, Koch TR. Prevalence of clinical thiamine deficiency in individuals with medically complicated obesity. Nutr Res. 2017 Jan;37:29-36.

31 Kerns JC, Arundel C, Chawla LS. Thiamin deficiency in people with obesity. Adv Nutr. 2015 Mar;6(2):147-53.

32 de Luis DA, Pacheco D, Izaola O, Terroba MC, Cuellar L, Cabezas G. Micronutrient status in morbidly obese women before bariatric surgery. Surg Obes Relat Dis. 2013 Mar-Apr;9(2):323-7.

33 Vitolo MR, Canal Q, Campagnolo PD, Gama CM. Factors associated with risk of low folate intake among adolescents. J Pediatr. 2006 Mar-Apr;82(2):121-6.

34 Coupaye M, Puchaux K, Bogard C, Msika S, Jouet $\mathrm{P}$, Clerici $\mathrm{C}$, et al. Nutritional consequences of adjustable gastric banding and gastric bypass: a 1-year prospective study. Obes Surg. 2009 Jan;19(1):56-65.

35 Wilson R, Willis J, Gearry R, Skidmore P, Fleming E, Frampton C, et al. Inadequate vitamin $\mathrm{C}$ status in prediabetes and type 2 diabetes mellitus: associations with glycaemic control, obesity, and smoking. Nutrients. 2017 Sep;9(9):1-15. 
36 Huerta MG, Roemmich JN, Kington ML, Bovbjerg VE, Weltman AL, Holmes VF, et al. Magnesium deficiency is associated with insulin resistance in obese children. Diabetes Care. 2005 May;28(5):1175-81.

37 Nielsen FH. Magnesium, inflammation, and obesity in chronic disease. Nutr Rev. 2010 Jun;68(6):333-40.

38 Yanoff LB, Parikh SJ, Spitalnik A, Denkinger B, Sebring NG, Slaughter P, et al. The prevalence of hypovitaminosis D and secondary hyperparathyroidism in obese Black Americans. Clin Endocrinol. 2006 May;64(5):5239.

39 Kimmons JE, Blanck HM, Tohill BC, Zhang J, Khan LK. Associations between body mass index and the prevalence of low micronutrient levels among US adults. MedGenMed. 2006 Dec;19;8(4):59.

40 Olusi SO. Obesity is an independent risk factor for plasma lipid peroxidation and depletion of erythrocyte cytoprotectic enzymes in humans. Int J Obes Relat Metab Disord. 2002 Sep;26(9):1159-64.

41 Furukawa S, Fujita T, Shimabukuro M, Iwaki M, Yamada Y, Nakajima Y, et al. Increased oxidative stress in obesity and its impact on metabolic syndrome. J Clin Invest. 2004 Dec; 114(12):1752-61.

42 Cembranel F, Hallal ALC, González-Chica DA, d'Orsi E. Relação entre consumo alimentar de vitaminas e minerais, índice de massa corporal e circunferência da cintura: um estudo de base populacional com adultos no Sul do Brasil. Cad Saúde Pública. 2017 Dec; 33(12):1-17.

43 Wassenaar TM, Zimmermann K. Lipopolysaccharides in food, food supplements, and probiotics: should we be worried? Eur J Microbiol Immunol. 2018 Sep;8(3):63-9.

44 Janczy A, Kochan Z, Małgorzewicz S. Endotoxemia and intestinal barrier disorders associated with overweight and obesity. Adv Microbiol. 2019 Jul;58(4):427-32.

45 Nicholson JK, Holmes E, Kinross J, Burcelin R, Gibson G, Jia W, et al. Host-gut microbiota metabolic interactions. Science. 2012 Jun; 336(6086):1262-7.

46 Kim B, Choi HN, Yim JE. Effect of diet on the gut microbiota associated with obesity. J Obes Metab Syndr. 2019 Dec;28(4):216-24.

47 Lazar V, Ditu LM, Pircalabioru GG, Picu A, Petcu L, Cucu N, et al. Gut microbiota, host organism, and diet trialogue in diabetes and obesity. Front Nutr. 2019 Mar;6(21):21.

48 Gérard P. Gut microbiota and obesity. Cell Mol Life Sci. 2016 Oct;73(1):147-62.
49 Żak-Gołąb A, Olszanecka-Glinianowicz M, Kocełak P, Chudek J. The role of gut microbiota in the pathogenesis of obesity. Postepy Hig Med Dosw. 2014 Jan;68:84-90.

50 Jumpertz R, Le DS, Turnbaugh PJ, Trinidad C, Bogardus C, Gordon JI, et al. Energy-balance studies reveal associations between gut microbes, caloric load, and nutrient absorption in humans. Am J Clin Nutr. 2011 May; 94(1):58-65.

51 Harris K, Kassis A, Major G, Chou CJ. Is the gut microbiota a new factor contributing to obesity and its metabolic disorders? J Obes. 2012 Jan;2012:879151-14.

52 Collado MC, Isolauri E, Laitinen K, Salminen S. Distinct composition of gut microbiota during pregnancy in overweight and normalweight women. Am J Clin Nutr. 2008 Oct; 88(4):894-9.

53 Gomes AC, Hoffmann C, Mota JF. The human gut microbiota: metabolism and perspective in obesity. Gut Microbes. 2018 May; 9(4):308-25.

54 Ley RE, Turnbaugh PJ, Klein S, Gordon JI. Microbial ecology: human gut microbes associated with obesity. Nature. 2006 Dec;444: $1022-3$.

55 Arumugam M, Raes J, Pelletier E, Le Paslier D, Yamada T, Mende DR, et al. Enterotypes of the human gut microbiome. Nature. 2011 Apr;473:174-80.

56 Brusaferro A, Cozzali R, Orabona C, Biscarin A, Farinelli E, Cavalli E, et al. Is it time to use probiotics to prevent or treat obesity? Nutrients. 2018 Dec;10(11):1613.

57 Abenavoli L, Scarpellini E, Colica C, Boccuto L, Salehi B, Sharifi-Rad J, et al. Gut microbiota and obesity: a role for probiotics. Nutrients. 2019 Sep;11(11):2690.

58 Benson AK, Kelly SA, Legge R, Ma F, Low SJ, $\mathrm{Kim} \mathrm{J}$, et al. Individuality in gut microbiota composition is a complex polygenic trait shaped by multiple environmental and host genetic factors. Proc Natl Acad Sci U S A. 2010 Nov;107(44):18933-8

59 Burcelin R, Serino M, Chabo C, Blasco-Baque V, Amar J. Gut microbiota and diabetes: from pathogenesis to therapeutic perspective. Acta Diabetol. 2011 Oct;48(4):257-73.

60 Nguyen NT, Varela JE. Bariatric surgery for obesity and metabolic disorders: state and art. Nat Rev Gastroenterol Hepatol. 2017 Mar; 14(3):160-9.

61 Shikora SA, Kim JJ, Tarnoff ME. Nutrition and gastrointestinal complications of bariatric surgery. Nutr Clin Pract. 2007 Feb;22(1): 29-40.
62 Shankar P, Boylan M, Sriram K. Micronutrient deficiencies after bariatric surgery. Nutrition. 2010 Nov-Dec;26(11-12):1031-7.

63 Guan B, Yang J, Chen Y, Yang W, Wang C. Nutritional deficiencies in Chinese patients undergoing gastric bypass and sleeve gastrectomy: prevalence and predictors. Obes Surg. 2018 Sep;28(9):2727-36.

64 Komorniak N, Szczuko M, Kowalewski B, Stachowska E. Nutritional deficiencies, bariatric surgery, and serum homocysteine level: review of current literature. Obes Surg. 2019 Aug;29:3735-42.

65 Billeter AT, Müller-Stich BP. [Metabolic surgery]. Chirurg. 2019 Feb;90(2):157-70.

66 Rohner F, Zimmermann M, Jooste P, Pandav C, Caldwell K, Raghavan R, et al. Biomarkers of nutrition for development - iodine review. J Nutr. 2014 Aug;144(8):1322S-42S.

67 Correia-Horvath JD, Dias de Castro ML, Kops N, Kruger-Malinoski N, Friedman R. Obesity coexists with malnutrition? Adequacy of food consumption by severely obese patients to dietary reference intake recommendations. Nutr Hosp. 2014 Feb;29(2):292-9.

68 Damms-Machado A, Friedrich A, Kramer KM, Stingel K, Meile T, Küper MA, et al. Preand postoperative nutritional deficiencies in obese patients undergoing laparoscopic sleeve gastrectomy. Obes Surg. 2012 Jun; 22(6):881-9.

69 Antoniewicz A, Kalinowski P, Kotulecka KJ, Kocoń P, Paluszkiewicz R, Remiszewski P, et al. Nutritional deficiencies in patients after Roux-en-Y gastric bypass and sleeve gastrectomy during 12-month follow-up. Obes Surg. 2019 Oct;29(10):3277-84.

70 Aigner E, Feldman A, Datz C. Obesity as an emerging risk factor for iron deficiency. $\mathrm{Nu}$ trients. 2014 Sep;6(9):3587-600.

71 Al-Mutawa A, Anderson AK, Alsabah S, AlMutawa M. Nutritional status of bariatric surgery candidates. Nutrients. 2018 Jan;10(1): $1-11$.

72 Maguire D, Talwar D, Shiels PG, McMillan D. The role of thiamine dependent enzymes in obesity and obesity related chronic disease states: a systematic review. Clin Nutr ESPEN. 2018 Jun;25:8-17.

73 Osland E, Powlesland H, Guthrie T, Lewis CA, Memon MA. Micronutrient management following bariatric surgery: the role of the dietitian in the postoperative period. Ann Transl Med. 2020 Mar;8(1):S9-16. 